\title{
PROGRAMAS PREVENTIVOS DE COMPORTAMENTOS ANTI-SOCIAIS: DIFICULDADES NA PESQUISA E NA IMPLEMENTAÇÃO
}

\author{
PREVENTIVE PROGRAMS OF ANTISOCIAL \\ BEHAVIORS: DIFFICULTIES IN THE \\ RESEARCH AND INTRODUCTION
}

\author{
Jocelaine Martins da SILVEIRA ${ }^{1}$ \\ Edwiges Ferreira de Mattos SILVARES ${ }^{2}$ \\ Simone Aparecida MARTON ${ }^{3}$
}

\begin{abstract}
RESUMO
Os programas preventivos de comportamentos anti-sociais em crianças e adolescentes são predominantemente marcados por dificuldades de duas ordens: a primeira é metodológica e dificuldades refere-seas à demonstração empírica de sua efetividade; enquanto que a segunda é prática e diz respeito aos obstáculos que o psicólogo costuma encontrar ao implementá-los em clínicas psicológicas públicas. Neste trabalho, sugerem-se alguns fatores de risco e de proteção sabidamente correlacionados ao desenvolvimento de comportamentos anti-sociais. Tais fatores podem nortear medidas proximais de suo efeito de programas preventivos daqueles comportamentos ao se delinear metodologia de pesquisa sobre o assunto. No que se refere à implementação de programas preventivos em clínicas-escola, levantam-se algumas alternativas para contornar obstáculos mais freqüentes. A oferta de serviços em locais mais acessíveis à população e o fornecimento de informações por meio de eventos de curta duração promovidos nas clínicas-escola são exemplos de alternativas.
\end{abstract}

Palavras-chave: Prevenção; comportamento anti-social; clínica-escola.

\footnotetext{
(1) Professora no Curso de Psicologia na Universidade Estadual de Londrina - Doutoranda pelo Instituto de Psicologia da USP - Endereço para correspondência: Departamento de Psicologia Geral e Análise do Comportamento Campus Universitário Caixa Postal 6001 - Cep: 86051-000 Londrina/PR.

(2) Professora Titular da Universidade de São Paulo.

(3) Graduanda da Universidade Estadual de Londrina.
} 


\begin{abstract}
The preventive programs of antisocial behavior of children and adolescents are prevailingly characterized by two kinds of difficulties: The first one is methodological and refers to the empirical evidence of its effectiveness; on the other hand, the second one is practical and deals with the obstacles the psychologist uses to find in order to introduce the above mentioned programs at public psychological clinics. The purpose of this paper is to suggest some well-known correlated risk and protection factors to the development of antisocial behaviors. Such factors may guide proximal measures of the effect of preventive programs of those behaviors by devising research methodology on the matter. To what concerns the introduction of preventive programs at school-clinics, some alternatives are raised to avoid the most frequent obstacles. The offer of services to the population at more convenient places and the delivery of information through short term events carried out at training clinics are examples of these alternatives.
\end{abstract}

Key words: Prevention; antisocial behavior; training clinics.

Identificar elementos relacionados ao desenvolvimento de problemas de comportamento em crianças e adolescentes tem sido a preocupação de muitos autores (Hallak, Hallak \& Golfeto, 1999; Hawkins, Catalano \& Miller, 1992; Bost, Vaughn, Washington, Cielinski \& Bradbard, 1998; Cowen, 1997; Forehand \& Wierson, 1993). Nos Estados Unidos, somente na década de 50 é que pesquisas sobre prevenção de desordens comportamentais em crianças e adolescentes começaram a ser realizadas. Segundo Durlak e Wells (1997), metade de todos os estudos controlados no campo apareceram a partir de 1980. Portanto, considerando que os efeitos de programas preventivos podem ser melhor observados ao longo do tempo, dispõem-se de um período relativamente curto, isto é, de aproximadamente duas décadas para que os efeitos daqueles estudos possam ser apreciados.

No Brasil, a despeito do reduzido número de investigações nessa área, há publicações que relatam estudos controlados em intervenções preventivas de desordens comportamentais na infância e na adolescência. Conte (1996) avaliou um programa de prevenção, aplicado a uma população de crianças e adolescentes em risco de delinqüência.

Convém distinguir os conceitos de "risco", "fatores de risco e de proteção" e "situação de risco" que serão empregados ao longo do texto. De acordo com Eisenstein e Souza (1993), risco é a probabilidade da ocorrência de algum evento indesejável. Fatores de risco são elementos com grande probabilidade de desencadear ou associar-se ao desencadeamento de um evento indesejado, não sendo considerados necessariamente o fator causal. Os fatores de proteção, por outro lado, são recursos pessoais ou sociais que atenuam ou neutralizam o impacto do risco. Já a expressão "situação de risco" pode ser entendida como uma circunstância que oferece risco a toda uma comunidade ou subgrupo social (Eisenstein \& Souza, 1993).

Quando são implementadas ações específicas para evitar o aparecimento de doenças e/ ou promover saúde, diz-se que a prevenção é primária (Eisenstein \& Souza, 1993). Detalhando este conceito, Durlak e Wells (1997) distinguem duas dimensões que o caracterizam: 1) a intervenção e 2) a forma como as populações são selecionadas. A intervenção pode incidir sobre as categorias: pessoal ou ambiental. 
Segundo esses autores, a seleção das populações sobre as quais a intervenção incide pode se dar de três maneiras: a primeira delas intervém em todos os membros de uma dada população, enquanto que a segunda intervém em grupos considerados de risco por problemas eventuais, mas que ainda não são disfuncionais como, por exemplo, filhos de pais que abusam do álcool. A terceira forma de selecionar a população focaliza aqueles que experimentam eventos vitais possivelmente estressores como, por exemplo, filhos de pais em processo de separação e alunos que se transferem de escolas.

Em geral, o efeito dos programas de prevenção se verifica por meio do aumento dos assim chamados fatores de proteção e, simultaneamente, da diminuição de fatores considerados de risco de surgimento de problemas futuros. Normalmente, são indicadores dessa mudança o aumento de comportamentos positivos ou aspectos do ambiente que enfraqueçam a probabilidade de resultados negativos ou que aumentem a possibilidade de resultados positivos.

\section{FATORES DE RISCO E DE PROTEÇÃO DE DESENVOLVIMENTO DE COMPORTAMENTOS ANTI-SOCIAIS EM CRIANÇAS}

Estudos recentes têm identificado fatores de risco de desenvolvimento de problemas de comportamentos anti-sociais infantis. Alguns estudos identificaram características demográficas correlacionadas a problemas de comportamentos anti-sociais da criança (Forehand \& Wierson,1993; Gavidia-Payne \& Stoneman, 1997; Garralda \& Bailey, 1988; Ghodian, Zajicek \& Wolking, 1984; Griest, Forehand, Wells \& Mcmanhon, 1980). Muitos desses problemas são merecedores de intervenções em um contexto bastante amplo, que transcende o ambiente clínico convencional.

O Transtorno da Conduta, de acordo com os critérios diagnósticos do DSM-IV (1994), é um padrão repetitivo e persistente de comportamento, no qual são violados os direitos básicos dos outros ou as normas sociais. O transtorno envolve violar regras, causar perdas ou danos a propriedades, defraudar ou furtar e causar danos físicos a pessoas ou a animais. O Transtorno Desafiador Opositivo, segundo o mesmo manual classificatório, é um padrão recorrente de comportamento negativista, desafiador, desobediente e hostil em relação a figuras de autoridade que acarreta um prejuízo significativo ao funcionamento social, acadêmico e ocupacional.

Sabe-se que certas características familiares fomentam o risco de desenvolvimento de Transtornos de Conduta na criança (WebsterStratton, 1990). Segundo Webster-Stratton (1998), a incidência do Transtorno Desafiador Opositivo e do Transtorno da Conduta em crianças pequenas é preocupante: estudos relataram que entre $7 \%$ a $25 \%$ de crianças pré-escolares atingem critérios diagnósticos de Transtorno Desafiador Opositivo, sendo que as taxas mais altas são encontradas em famílias de baixa renda (Webster-Stratton, 1998).

De acordo com Webster-Stratton (1998), esses dados são especialmente preocupantes uma vez que os problemas de comportamentos anti-sociais observados nos primeiros anos de vida, tais como altas taxas de comportamento opositor, agressivo e de desobediência em idades pré-escolares, são estáveis e predizem não só problemas escolares, mas também sérios problemas de saúde e problemas comportamentais na adolescência, como abuso de drogas, depressão, delinqüência juvenil e abandono da escola (Egeland, Kalkoske, Gottesman \& Erickson, 1990).

Alguns fatores de risco de desenvolvimento de problemas de comportamento anti-social podem ser mais prontamente notados na própria criança. Dificuldades acadêmicas que acontecem precocemente na vida escolar da criança são associadas a comportamentos anti-sociais (Schonfeld, Shaffer, O'Connor \& Portnoy, 1988). Habilidades sociais e de resolução de problemas 
deficitárias são também relacionadas àqueles comportamentos (Webster-Stratton, 1998).

Alguns estudos indicaram que variáveis presentes no contexto familiar podem favorecer o desenvolvimento de comportamentos anti-sociais nos filhos (Patterson, Debaryshe \& Ramsey, 1989). As características familiares correlacionadas ao desenvolvimento de problemas de comportamento anti-social na criança são: baixa renda, baixa escolaridade, gravidez na adolescência, isolamento, alto grau de estresse, pais solteiros, doença psiquiátrica parental, história parental criminal e abuso de substâncias, alto grau de conflitos conjugais e depressão (Webster-Stratton, 1990). Adicionalmente, quando há inconsistência nas práticas disciplinares parentais, abuso físico, hostilidade e crítica por parte dos pais, é alto o risco de desenvolvimento de comportamentos anti-sociais nas crianças (Patterson \& Stouthamer-Loeber, 1984).

Foram identificados ainda como fatores relacionados ao desenvolvimento de tais comportamentos, pais não engajados nas experiências escolares de seus filhos e que os provêem de pouca estimulação cognitiva (Hawkins, Catalano \& Miller, 1992; Webster-Stratton, 1998). Patterson, Derbayshe e Ramsey (1989) apontam, em seu estudo, que pais de famílias de classe média tendem a usar métodos mais racionais e psicológicos de disciplina, dando aos filhos mais chance de escolha e autodireção; tendem a ter padrões mais igualitários entre si; expressam afeto positivo mais freqüentemente; verbalizam mais e dão mais apoio ao desenvolvimento cognitivo e acadêmico de seus filhos, quando comparados a pais de famílias com renda inferior. Pais de classe econômica baixa tendem a usar mais a disciplina física, ter estilos de paternidade mais autoritários e controladores e engajar-se menos freqüentemente em interações verbais, estimulação cognitiva, monitoramento e supervisão acadêmica. Por fim, convém mencionar que há evidências de que o risco de a criança desenvolver comportamentos anti-sociais aumenta exponencialmente com a exposição a cada fator de risco adicional (Coie, Watt, West, Hawkins,
Asarnow, Markman, Ramey, Shure \& Long, 1993).

A Tabela 1 apresenta uma lista de fatores de risco de desenvolvimento de problemas de comportamento anti-social freqüentemente citados na literatura e os respectivos fatores de proteção.

É possível notar na Tabela 1 que alguns fatores de risco demandam intervenções em contextos sociais amplos, enquanto que outros podem sofrer mudanças mediante intervenções realizadas com os pais e/ou com estes e seus filhos no âmbito da família. Quando a intervenção se dá no contexto da família, seus efeitos são mais facilmente perceptíveis e/ou verificáveis por meio de medidas ditas proximais, embora possam estender-se ao longo do desenvolvimento da criança, podendo também ser verificados em aferições distais. Isto é, ao se intervir aumentando fatores de proteção e enfraquecendo fatores de risco de desenvolvimento de comportamentos anti-sociais, promove-se a prevenção de diversos outros problemas comportamentais subseqüentes na vida da criança.

Os fatores listados na Tabela 1 exemplificam possíveis parâmetros de objetivos proximais para programas preventivos. Ou seja, embora programas preventivos visem promover resultados em um futuro remoto, suas intervenções redundam em resultados positivos imediatos para a população atendida. Clínicos e pesquisadores podem valer-se deles para delinear medidas de avaliação da efetividade de programas preventivos.

\section{MEDIDAS PROXIMAIS E DISTAIS NA PESQUISA DA EFETIVIDADE DE PROGRAMAS PREVENTIVOS}

Segundo Durlak e Wells (1997), os programas preventivos devem ser avaliados de acordo com seus objetivos proximais e distais. Porém, a demonstração empírica da efetividade de programas preventivos costuma ser limitada por diversos fatores. Um deles é o desconhecimento acerca do curso de desenvolvimento 
Tabela 1. Fatores de risco e de proteção relacionados ao desenvolvimento de comportamentos antisociais em crianças.

\begin{tabular}{|c|c|c|c|}
\hline Fator de risco & $\begin{array}{l}\text { Em quem se } \\
\text { pode notar }\end{array}$ & $\begin{array}{l}\text { Fator de proteção que se pode } \\
\text { fomentar }\end{array}$ & $\begin{array}{l}\text { Em quem a intervenção } \\
\text { poderá incidir }\end{array}$ \\
\hline Atraso de linguagem & Criança & $\begin{array}{l}\text { Habilidades parentais de estimulação } \\
\text { cognitiva, aliadas a interações afetivas } \\
\text { com a criança. }\end{array}$ & Pais e Criança \\
\hline Atrasos cognitivos & Criança & $\begin{array}{l}\text { Habilidades parentais de estimulação } \\
\text { cognitiva. }\end{array}$ & Pais e Criança \\
\hline $\begin{array}{l}\text { Habilidades de leitura } \\
\text { deficitárias }\end{array}$ & Criança & $\begin{array}{l}\text { Engajamento parental em atividades } \\
\text { acadêmicas, aliado à estimulação } \\
\text { cognitiva }\end{array}$ & Pais e Criança \\
\hline $\begin{array}{l}\text { Habilidades sociais } \\
\text { deficitárias }\end{array}$ & Pais & $\begin{array}{l}\text { Aumento da competência social pa- } \\
\text { rental e engajamento parental em } \\
\text { atividades comunitárias. }\end{array}$ & Pais \\
\hline $\begin{array}{l}\text { Habilidades de } \\
\text { resolução de } \\
\text { problemas deficitárias }\end{array}$ & Pais & $\begin{array}{l}\text { Competência parental para resolução } \\
\text { de problemas }\end{array}$ & Pais \\
\hline $\begin{array}{l}\text { Baixa renda. História } \\
\text { criminal. Baixa escola- } \\
\text { ridade }\end{array}$ & Família & $\begin{array}{l}\text { Esforços em contextos sociais mais } \\
\text { amplos }\end{array}$ & Família \\
\hline $\begin{array}{l}\text { Gravidez na } \\
\text { adolescência }\end{array}$ & Mãe & $\begin{array}{l}\text { Esforços em contextos sociais mais } \\
\text { amplos }\end{array}$ & Família \\
\hline Isolamento & Família & $\begin{array}{l}\text { Esforços em contextos sociais mais } \\
\text { amplos }\end{array}$ & Família \\
\hline $\begin{array}{l}\text { Altos níveis de } \\
\text { estresse }\end{array}$ & Mãe & $\begin{array}{l}\text { Expansão e fortalecimento de redes } \\
\text { de apoio. Afiliação religiosa. Fortale- } \\
\text { cimento de estratégias de coping. }\end{array}$ & Mãe \\
\hline Pais solteiros & Pais & $\begin{array}{l}\text { Expansão e fortalecimento de redes } \\
\text { de apoio. Afiliação religiosa. }\end{array}$ & Pais \\
\hline Doença psiquiátrica & Pais & $\begin{array}{l}\text { Esforços em contextos sociais mais } \\
\text { amplos. Desenvolvimento na criança } \\
\text { de habilidades de proteger-se. }\end{array}$ & Pais e Criança \\
\hline Depressão & Mãe & $\begin{array}{l}\text { Desenvolvimento de repertório com- } \\
\text { portamental incompatível com de- } \\
\text { pressão. }\end{array}$ & Mãe \\
\hline
\end{tabular}




\begin{tabular}{|c|c|c|c|}
\hline Fator de risco & $\begin{array}{l}\text { Em quem se } \\
\text { pode notar }\end{array}$ & $\begin{array}{l}\text { Fator de proteção que se pode } \\
\text { fomentar }\end{array}$ & $\begin{array}{l}\text { Em quem a intervenção } \\
\text { poderá incidir }\end{array}$ \\
\hline Abuso de substâncias & Pais & $\begin{array}{l}\text { Esforços em contextos mais amplos. } \\
\text { Desenvolvimento na criança de compe- } \\
\text { tências sociais, entre outras. }\end{array}$ & Pais e Criança \\
\hline $\begin{array}{l}\text { Inconsistência na } \\
\text { disciplina }\end{array}$ & Pais & $\begin{array}{l}\text { Desenvolvimento de habilidades espe- } \\
\text { cíficas de cuidado. }\end{array}$ & Pais \\
\hline Hostilidade e crítica & Pais & $\begin{array}{l}\text { Desenvolvimento de habilidades } \\
\text { parentais específicas. }\end{array}$ & Pais \\
\hline $\begin{array}{l}\text { Habilidades deficitá- } \\
\text { rias de cuidado do } \\
\text { primogênito }\end{array}$ & Mãe & $\begin{array}{l}\text { Desenvolvimento de habilidades } \\
\text { maternas específicas }\end{array}$ & Mãe \\
\hline Déficit em autocontrole & Criança & Desenvolvimento de autocontrole & Criança \\
\hline Problemas de conduta & Pais & $\begin{array}{l}\text { Treinamento em habilidades sociais } \\
\text { incompatíveis }\end{array}$ & Pais \\
\hline Abuso físico & Pais & $\begin{array}{l}\text { Desenvolvimento de habilidades } \\
\text { parentais específicas. } \\
\text { Desenvolvimento na criança de } \\
\text { habilidades de proteger-se dosadultos. }\end{array}$ & Pais e Criança \\
\hline
\end{tabular}

preciso da maioria dos problemas da infância. Outro aspecto que dificulta a demonstração empírica da efetividade de programas preventivos refere-se à multideterminação dos comportamentos-problema (Durlak \& Wells, 1997). Isto implica a necessidade de que os programas sejam complexos e com múltiplos componentes. De qualquer modo, essas dificuldades não devem desencorajar as pesquisas na área. Antes, elas podem incentivar a busca de ajustamentos metodológicos.

\section{ALTERNATIVAS PARA IMPLEMENTAÇÃO DE PROGRAMAS PREVENTIVOS EM CLÍNICAS-ESCOLA}

O atendimento psicológico infantil, especialmente às camadas populacionais de menor poder aquisitivo, tem tradicionalmente seguido um mesmo caminho, qual seja: da escola para as clínicas públicas de atendimento psicológico. Os agentes sociais da escola (professores, assistentes sociais, diretores e outros) percebem dificuldades de caráter acadêmico ou outro qualquer nas crianças e encaminham-nas para psicólogos ou para as clínicas-escola de Psicologia. As clínicas-escola localizam-se em centros universitários e foram criadas para proporcionar estágio aos alunos de cursos de graduação em Psicologia, sendo supervisionados por professores (Silvares, 2000).

Os resultados obtidos por estudos brasileiros de caracterização da clientela infantil em clínicas-escola de Psicologia (Lopez, 1983; Santos, 1990) têm corroborado e evidenciado a inadequação da referida forma de encaminhamento. O procedimento usual de encaminhamento psicológico para terapia infantil tem inflado o 
número de crianças que aguardam atendimento em lista de espera nas clínicas-escola.

As clínicas-escola brasileiras estão localizadas, em geral, em pontos distantes das moradias da clientela, isto é, situam-se nos centros universitários e são procuradas predominantemente por pessoas de nível sócio-econômico baixo. Assim, o deslocamento até as mesmas torna-se financeiramente custoso para a clientela, que muitas vezes desiste do atendimento enquanto aguarda em lista de espera (Silvares, 1993; 2000).

A necessidade de mudanças nas práticas das clínicas-escola brasileiras vem sendo indicada há bastante tempo (Macedo, 1984). Há, entretanto, poucos trabalhos realizados para alterar a via de encaminhamento aqui discutida. Quando as intervenções têm caráter preventivo, a adesão da clientela tende a ser ainda menor.

De que modo atrair para as clínicas-escola pais de crianças que "ainda" não apresentam problemas comportamentais? Os pais tendem a procurar o serviço psicológico quando algum problema comportamental já está instalado no repertório da criança. Sabidamente, a renda média familiar das pessoas que procuram o atendimento em clínicas-escola é baixa e o custo do deslocamento até a clínica, muitas vezes, somente se justifica caso o problema no comportamento da criança incomode os pais ou a escola.

Por outro lado, quando os pais procuram atendimento preventivo em clínicas psicológicas públicas, os mesmos acabam sendo preteridos na fila de espera, em conseqüência da urgência de muitos outros casos, já que a maioria das clínicas apresenta problemas de demanda excessiva.

Aparentemente, esses obstáculos podem ser superados pelo modo de se recrutar os pais para os programas e pela forma de intervir para promover prevenção de problemas comportamentais em crianças. Os estagiários poderiam se deslocar do campus universitário para locais mais estratégicos, atendendo a comunidade por meio de escolas, postos de saúde ou instituições religiosas em bairros da cidade. Conte (1996) adotou essa estratégia com sucesso em um programa de prevenção da delinqüência.

São necessárias modalidades de atendimento preventivo de desenvolvimento de desordens de conduta infantil que atendam aos pais no momento em que estes procuram serviços imprescindíveis para a saúde da criança. Assim, os pais poderiam receber orientações sobre como proceder com seus filhos, no mesmo local em que consultam um profissional de obstetrícia e/ou pediatria, por exemplo. Em um estudo de revisão de programas preventivos de problemas comportamentais em crianças, Durlak e Wells (1997) verificaram que os programas eficazes oferecidos às mães de primeiro filho, em geral, combinam uma variedade de serviços que, eventualmente, estendem-se às crianças.

O fornecimento de informações básicas sobre como educar a criança é o ponto central da intervenção preventiva viável no âmbito psicológico, de tal modo que os pais se tornem conscientes de como seu comportamento influencia o comportamento da criança. Desse modo, atrair os pais para participar de palestras, debates e workshops que informam e treinam habilidades para a educação de seus filhos pode contribuir com o fornecimento de informações pertinentes. Esses encontros poderiam tratar de temas básicos, porém, imprescindíveis para o bom relacionamento pais-criança, tais como: adotar práticas disciplinares coerentes (Fauber \& Long, 1991); evitar hostilidade aberta interparental na presença da criança (Emery, 1982); desenvolver repertório de monitoramento da criança (Webster-Stratton, 1998); desenvolver repertório para promover obediência na criança (Rayfield, Monaco \& Eyberg, 1998), entre outros.

Silvares (2000) relatou um atendimento a crianças em grupos recreativos de lista de espera em clínicas-escola. $O$ atendimento mostrou-se efetivo para evitar desistências antes que o tratamento se iniciasse. Os grupos recreativos têm garantida a permanência da criança na lista de espera para atendimento e têm sido úteis 
para a identificação de padrões comportamentais merecedores de atenção clínica, possibilitando um avanço na fase de avaliação, quando o atendimento tem início.

A oferta de serviços na modalidade de grupos abertos é uma outra possibilidade. Usualmente, realizam-se intervenções em grupos fechados com, em média, dez encontros. Porém, ocorrem muitos abandonos prematuros do tratamento. Alguns desses abandonos se dão quando os pais já obtiveram ganhos com o tratamento e julgam não necessitarem mais do atendimento psicológico. Esses abandonos devem ser vistos em um contexto no qual o custo do deslocamento do cliente até a clínica é importante, considerando a renda familiar média das pessoas atendidas. Em razão disso, seria interessante a existência de grupos abertos que permitissem a realização de encaminhamento e de aconselhamento breve. Assim, os pais poderiam comparecer e receber orientações e/ou treinamento breve. Os fatores de risco e proteção aqui listados podem constituir a temática potencial de palestras, cursos e programas de orientação breve.

Por último, o uso de instrução e treinamento por meio de videoteipe, com vinhetas que ilustram situações em que comumente os pais encontram dificuldades em lidar com seus filhos, também pode ser apropriado para atender a clientela de pais em clínicas-escola nas condições descritas acima.

Concluindo, o presente artigo sugere que os fatores de risco e de proteção de desenvolvimento de comportamentos anti-sociais poderiam nortear delineamentos de pesquisa de programas preventivos desses comportamentos. Os mesmos fatores poderiam também inspirar a tônica dos serviços prestados à comunidade nas clínicas-escola.

\section{REFERÊNCIAS BIBLIOGRÁFICAS}

AMERICAN Psychiatric Association (1994). Diagnostic and statistical manual of mental disorders, DSM-IV. $4^{\mathrm{a}}$ edição. Washington D.C.: American Psychiatric Press.

BOST, K.K.; Vaughn, B. E.; Washington, W. N.; Cielinski, K.L.; Bradbard, M.R. (1998). Social competence, social support, and attachment: demarcation of construct domains, measurement, and paths of influence for preschool children attending head start. Child development, 69 (1), 192-218.

COIE, J. D.; Watt, N.F.; West, S.G.; Hawkins, J. D.; Asarnow, J. R.; Markman, H. J.; Ramey, S. L.; Shure, M. B. \& Long, B. (1993). The science of prevention: A conceptual framework and some directions for a national research program. American Psychologist, 48,1013-1022.

CONTE, F. C. de S. (1996). Pesquisa e Intervenção Clínica em Comportamento Delinqüente numa Comunidade Pobre. Tese de Doutorado, Instituto de Psicologia, Universidade de São Paulo, São Paulo.

COWEN, E. L. (1997). The coming of age of primary prevention: Comments on Durlak and Well's meta-analysis. American Journal of Community Psychology, 25, 153-167.

DURLAK, J.A. \& Wells, A. M. (1997). Primary Prevention Mental Health Programs for Children and Adolescents: A Meta-Analytic Review. American Journal of Community Psychology, 25 (2), 115-152.

EGELAND, B.; Kalkoske, M.; Gottesman, N. \& Erickson, M.F. (1990). Preschool behavior problems: stability and factors accounting for change. Journal of Child Psychology and Psychiatry, 31, 891-909.

EISENSTEIN, E \& Souza, R. P. de. (1993). Situações de risco à saúde de crianças e adolescentes. Petrópolis, RJ: Vozes.

EMERY, R. E. (1982). Interparental conflict and children of discord and Divorce. Psychological Bulletin, 92 (2), 310-330.

FAUBER, R. L. \& Long, N. (1991). Children in Context: The role of the family in Child Psychotherapy. Journal of Consulting and Clinical Psychology, 59 (6), 813-820. 
FOREHAND, R. \& Wierson, M. (1993). The role of developmental factors in planning behavioral interventions for children: Disruptive behavior as an example: Behavior Therapy, 24, 117-141.

GARRALDA, M.E. \& Bailey, D. (1988). Child and Family Factors Associated with referral to child Psychiatrists. British journal of Psychiatry, 153, 81-89.

GAVIDIA-PAYNE, S. \& Stoneman, Z. (1997). Family predictors of maternal and parental involvement in programs for young children with disabilities. Child Development, 68 (4), 701-717.

GHODIAN, M.; Zajicek, E. \& Wolking, S. (1984) A longitudinal study of maternal depression and child behavior problems. Journal of Child Psychology and Psychiatry, 25, 91-109.

GRIEST, D. L.; Forehand, R.; Wells, K.C. \& Mcmanhon, R. J. (1980). An examination of differences between nonclinic and behavior problem children clinic-referred children and their mothers. Journal of Abnormal Psychology, 89, 497-500.

HALLAK, L. R. de; Hallak, J. E. C. \& Golfeto, J. H. (1999). O estresse materno e os fatores de risco em crianças com distúrbios psiquiátricos. Pediatria Moderna, 35 (9), 731-740.

HAWKINS, J. D.; Catalano, R. F. \& Miller, Y. (1992). Risk and protective factors for alcohol and other drug problems in adolescence and early adulthood: Implications for substance abuse prevention. Psychological Bulletin, 112, 64-105.

LOPEZ, M. A. (1983). Características da clientela de clínicas-escola de Psicologia em São Paulo. Arquivos Brasileiros de Psicologia, 35, 78-92.

MACEDO, R. M. (1984). Psicologia e Instituição: Novas formas de atendimento. São Paulo: Ed. Cortez.
PATTERSON, G. R.; Debaryshe, B. D. \& Ramsey, E. (1989). A developmental Perspective on Antisocial Behavior. American Psychologist. 44 (2), 329-335.

PATTERSON, G. R. \& Stouthamer-Loeber, M. (1984). The correlation of family management practices and delinquency. Child Development, 55, 1299-1307.

RAYFIELD, A.; Monaco, L. \& Eyberg, S. (1998). Parent-child interaction therapy with oppositional children: Review and clinical strategies. Em: S. Russ e T. Ollendick (Org.). Handbook of psychotherapies with children and families. Nova York: Plenum.

SANTOS, M. A. (1990). Caracterização da clientela de uma clínica psicológica da Prefeitura de São Paulo. Arquivos Brasileiros de Psicologia, 41, 70-94.

SCHONFELD, I. S.; Shaffer, D.; O'Connor, P. \& Portnoy, S. (1988). Conduct disorder and Cognitive functioning: Testing three causal hypotheses. Child Development, 59, 993-1007.

SILVARES, E. F. de M. (1993). O papel preventivo das clínicas-escola de psicologia em seu atendimento a crianças. Temas em Psicologia, 2, 87-97.

SILVARES, E. F. de M. (2000). Invertendo o caminho tradicional do atendimento psicológico em uma clínica-escola brasileira. Estudos de Psicologia (RN), 5 (1), 149-180.

WEBSTER-STRATTON, C. (1998) Preventing conduct problems in head start children: strengthening parenting competencies. Journal of Consulting and Clinical Psychology, 66 (5), 715-730.

WEBSTER-STRATTON, C. (1990). Stress: A potential disruptor of parent perceptions and family interactions. Journal of Consulting and Clinical Psychology, 19, 302-312.

Recebido para publicação em 5 de fevereiro de 2002 e aceito em 18 de agosto de 2003. 
